\title{
Article \\ Morphological Change in the Biceps Brachii Muscles during Shoulder Rotation: A Cadaver Study
}

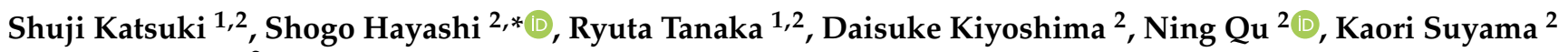 \\ and Kou Sakabe ${ }^{2}$ \\ 1 Department of Rehabilitation, Kanto Rosai Hospital, 1-1 Kizukisumiyoshi-cho, Nakahara-ku, Kawasaki-shi, \\ Kanagawa 211-8510, Japan; katsuki@kantoh.johas.go.jp (S.K.); ryuta-tanaka@kantoh.johas.go.jp (R.T.) \\ 2 Department of Anatomy, Division of Basic Medical Science, Tokai University School of Medicine, \\ 143 Shimokasuya, Isehara-shi, Kanagawa 259-1193, Japan; kiyoshima@tokai.ac.jp (D.K.); \\ quning@tokai.ac.jp (N.Q.); suyama@is.icc.u-tokai.ac.jp (K.S.); sakabek@tokai-u.jp (K.S.) \\ * Correspondence: sho5-884@umin.ac.jp; Tel.: +81-463-93-1121
}

check for

updates

Citation: Katsuki, S.; Hayashi, S.; Tanaka, R.; Kiyoshima, D.; Qu, N.; Suyama, K.; Sakabe, K.

Morphological Change in the Biceps Brachii Muscles during Shoulder Rotation: A Cadaver Study. Appl. Sci. 2021, 11, 9262. https://doi.org/ 10.3390/app11199262

Academic Editors: Nikolaos Zaras and Spyridon Methenitis

Received: 6 September 2021

Accepted: 2 October 2021

Published: 6 October 2021

Publisher's Note: MDPI stays neutral with regard to jurisdictional claims in published maps and institutional affiliations.

Copyright: (c) 2021 by the authors. Licensee MDPI, Basel, Switzerland. This article is an open access article distributed under the terms and conditions of the Creative Commons Attribution (CC BY) license (https:/ / creativecommons.org/licenses/by/ $4.0 /)$.

\begin{abstract}
In the literature, the passive movement of the biceps brachii during shoulder motion is unclear. This study investigated the passive movement of the long head (LHBB) and short head (SHBB) of the biceps brachii during shoulder rotation. We included 18 shoulders from 15 cadavers. At three shoulder positions (internal rotation $[\mathrm{IR}] 20^{\circ}, 0^{\circ}$, external rotation [ER] $20^{\circ}$ ), the three sides of the triangle consisting of the confluence of the SHBB and LHBB, coracoid processes, and lesser tubercles were measured. The confluence angle of the LHBB and SHBB (Angle A) was calculated using the cosine theorem. The mean values for Angle A and standardized three sides of the triangle were statistically compared among the three shoulder positions. Angle A values were $13.5^{\circ} \pm 7.6^{\circ}$ at IR2 $0^{\circ}, 15.2^{\circ} \pm 5.8^{\circ}$ at $0^{\circ}$, and $15.7^{\circ} \pm 4.3^{\circ}$ at ER2 $0^{\circ}$ with no significant difference $(p=0.096)$. The lengths of all three sides and Angle A were the smallest at IR2 $0^{\circ}$ and highest at ER2 $0^{\circ}$. All the three sides revealed significant differences among the three shoulder positions $(p<0.0001)$. These results imply that morphological changes of the biceps brachii occur during shoulder rotation. These morphological changes in biceps brachii may contribute to shoulder joint stabilization by adjusting the forces the act on the tendons of both heads.
\end{abstract}

Keywords: anatomy; biceps brachii; cadaver; coracoid process fracture; physical therapy; rehabilitation; rotation; shoulder; stabilizing system; surgery; tendon

\section{Introduction}

The biceps brachii muscle is a powerful supinator, especially in rapid or resisted movements. It flexes the elbow joint, most effectively with the forearm supinated [1]. The biceps brachii is composed of a long and a short head. The long head of the biceps brachii (LHBB) originates from the supraglenoid tubercle of the scapula. In contrast, the short head of the biceps brachii (SHBB) originates from the tip of the coracoid process (CP) and inserts into the radial tuberosity and the fascia of the forearm [2]. The LHBB passes downward through the intertubercular sulcus (IS) between the two humeral tubercles $[3,4]$. Two tendons, which lie side-by-side, lead into elongated bellies and are separated within approximately $7 \mathrm{~cm}$ to the elbow joint [1].

The role of the biceps brachii in shoulder joint rotation has been studied, mostly focusing on the function of the LHBB [5-7]. In a cadaver study, Itoi et al. [5] indicated that the loaded LHBB provided a stabilizing function to the humeral head in all directions and more importantly, in the anterior-posterior directions in the hanging arm position. Some clinical studies have also suggested that the LHBB acts as a stabilizer in cases of rotator cuff tears [8-11]. Given these observations, the LHBB is thought to contribute to the stability of the shoulder joint. In contrast, little is known on how the SHBB affects the shoulder joint. 
Considering the attachments of the LHBB and SHBB, the biceps brachii muscle seems to change its shape passively during rotation of the shoulder joint. During external rotation (ER) of a normal shoulder joint, the intertubercular sulcus (IS) moves outward. Because the LHBB passes through the IS, it moves outward with ER of the shoulder joint; vice versa, upon internal rotation (IR), the IS and LHBB move inward. Since the rotation of the shoulder joint mainly occurs at the glenohumeral joint, the movement of the humerus is greater than that of the scapula. Hence, during rotation of the shoulder, the movement of the CP must be smaller than that of the IS. In other words, the LHBB and SHBB may passively move apart during ER and move closer during IR. Concomitantly, the angle of the confluence of the LHBB and SHBB may also increase or decrease, respectively. However, it is not clear how the LHBB and SHBB passively change their morphology during shoulder motion.

In the present study, we aimed to investigate the passive morphological changes of the biceps brachii muscle during rotation of the shoulder joint, with a special focus on the angle between the LHBB and SHBB.

\section{Materials and Methods}

\subsection{Subjects}

In this study, we analyzed 18 shoulders of 15 cadavers (males: 5, females: 10, age: $84.6 \pm 11.6$ years) donated to Tokai University School of Medicine and used for dissection practice between April 2017 and July 2020 (Table 1). The cadavers were fixed with 10\% formaldehyde. In preparation for the measurements, a gross dissection was performed. The cadaver was placed in a supine position. The skin, fat, and subcutaneous tissue around the chest and the shoulder joint were removed, and the anterior shoulder joint structures were carefully observed. Next, the origin of the deltoid and pectoralis major muscles was excised and inverted, and the LHBB and SHBB were observed under direct vision.

Table 1. Subject data.

$\begin{array}{cc}\text { Subject } & 18 \text { shoulders } \\ \text { Sex } & \text { Males: 5; Females: } 10 \\ \text { Age (years) } & 84.6 \pm 11.6 \\ \text { Length of humerus }(\mathrm{mm}) & 284.7 \pm 15.2\end{array}$

\subsection{Data Acquisition and Analysis}

The conditions for the inclusion of the cadavers in this experiment were as follows: (1) no damage or invasion of the biceps brachii muscle; (2) a rotational range of motion of the shoulder joint from IR $20^{\circ}$ to ER $20^{\circ}$, and flexion range of motion of the elbow joint of approximately $90^{\circ}$; and (3) no clinical history of fracture of the shoulder joint. Twelve shoulders of the cadavers that did not meet these conditions were excluded from this study.

For the measurements, the upper limb was placed at the side, and the elbow joint was flexed approximately $90^{\circ}$. Three shoulder positions were measured, which were as follows: $\operatorname{IR} 20^{\circ}, 0^{\circ}$, and ER2 $0^{\circ}$. To eliminate the stretching effect of the measurements on the biceps brachii muscle, the measurements were taken only once.

In each of the three shoulder positions, three points were set: $\mathrm{A}$, confluence of the LHBB and SHBB; B, lateral side of the SHBB at the top of the CP level; and C, medial side of the LHBB tendon at the top of the lesser tubercle (LT) level. The distances of Sides AB, AC, and $B C$ were measured. Based on these three sides, a virtual triangle was created. Angle A was formed by Sides AB and AC as the angle between LHBB and SHBB. To calculate for Angle A using the cosine theorem, we measured the distance of the three sides (AB, AC, and $\mathrm{BC}$ ) of this triangle (Figure 1). 


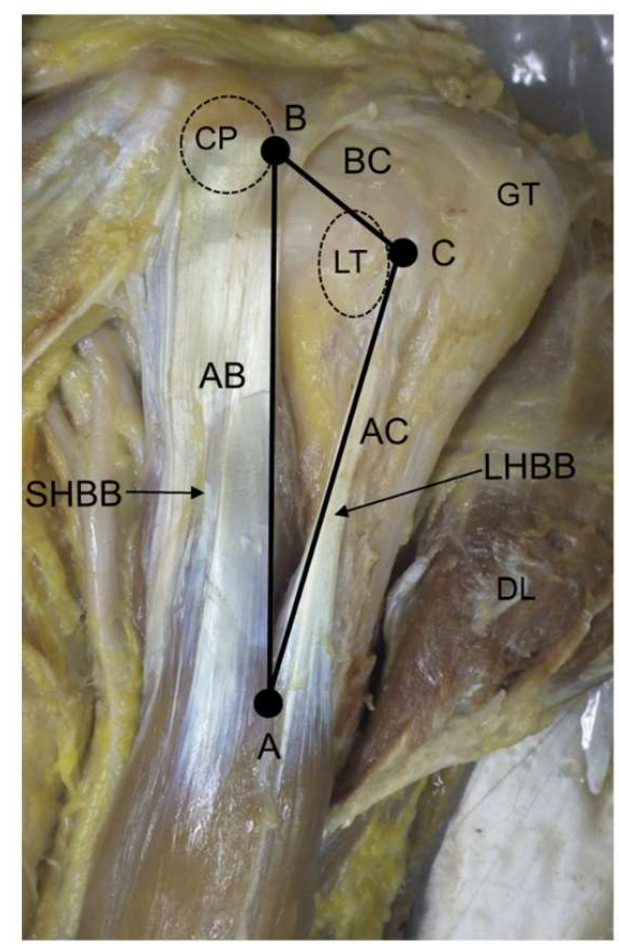

Figure 1. Anterior view of the left shoulder joint. A: Confluence between the LHBB and SHBB. B: Lateral side of the SHBB at the top of the CP level. C: Medial side of the LHBB tendon at the top of the lesser tubercle level. CP, coracoid process; DL, deltoid; GT, greater tubercle; LHBB, long head of biceps brachii; LT, lesser tubercle; SHBB, short head of biceps brachii.

\subsection{Statistical Analysis}

All statistical analyses were performed with JMP ${ }^{\circledR}$ (Version 15.2.1, SAS Institute Inc., Cary, NC, USA) and Prism 9 for macOS (Version 9.0.0, GraphPad Software, San Diego, CA, USA). The measured lengths of Sides $A B, A C$, and $B C$ were standardized by dividing these lengths by the humeral length. The LHBB and SHBB have different origins. Therefore, the measurements of each side were standardized by the humeral length, which is close to the length of the biceps brachii. The humeral length was defined as the length between the lateral side of the acromion and lateral epicondyle of the humerus. Bartlett's tests were performed to examine the variance between the three positions. Repeated measures analyses of variance (ANOVA) using the Greenhouse-Geisser correction, and Tukey's multiple comparisons tests were used to investigate the differences in mean values between the three positions of the shoulder for Angle A and standardized Sides AB, AC, and BC. The significance level was less than $5 \%$ for each analysis. Gender differences were investigated using Student's t-test.

This report complies with the research guidelines of the Japanese Association of Anatomists. The cadavers used in this study were donated (Tokai Daigaku Kentai No Kai) for education or research. Informed consent was obtained from the antemortem person, Tokai Daigaku Kentai No Kai. This study was conducted under the approval of the Ethics Committee of Tokai University School of Medicine (Ethics Review Number 17R-307).

\section{Results}

Table 2 summarizes the side lengths and the calculated angles. Data are presented as mean \pm standard deviation. All the three sides and Angle A were the lowest at IR20 and the highest at $\mathrm{ER} 20^{\circ}$. While there was no significant difference in Angle $\mathrm{A}(p=0.096)$, the length of all the sides $(\mathrm{AB}, \mathrm{AC}$, and $\mathrm{BC})$ showed significant differences among the three shoulder positions (Figure 2). Angle A values were $13.5^{\circ} \pm 7.6^{\circ}$ at IR20 $0^{\circ}, 15.2^{\circ} \pm 5.8^{\circ}$ at $0^{\circ}$, and $15.7^{\circ} \pm 4.3^{\circ}$ at ER2 $20^{\circ}$. In cases with large angles at IR, Angle A tended to decrease 
towards ER, whereas in the presence of small angles at IR, it tended to increase towards ER (Figure 3). Bartlett's test results showed that for Angle A, there was a trend towards a variability in the values at the three shoulder positions $(p=0.07)$ (Figure 4$)$. There was no statistically significant difference between males and females in the three shoulder positions of standardized Side AB, Side AC, Side BC, and Angle A (Table 3).

Table 2. Measurements of Sides AB, AC, and BC, and Angle A according to the three shoulder positions.

\begin{tabular}{cccc}
\hline & IR20 $^{\circ}$ & $\mathbf{0}^{\circ}$ & ER20 $^{\circ}$ \\
\hline Angle A [degree] & $13.5 \pm 7.6$ & $15.2 \pm 5.8$ & $15.7 \pm 4.3$ \\
Side AB [mm] & $68.7 \pm 17.4$ & $83.6 \pm 15.2$ & $93.1 \pm 12.2$ \\
Side AC [mm] & $60.7 \pm 17.8$ & $74.8 \pm 14.3$ & $83.2 \pm 12.8$ \\
Side BC [mm] & $17.3 \pm 5.3$ & $22.8 \pm 6.1$ & $26.2 \pm 6.6$ \\
\hline
\end{tabular}

Data are presented as mean \pm standard deviation. ER, external rotation; IR, internal rotation.

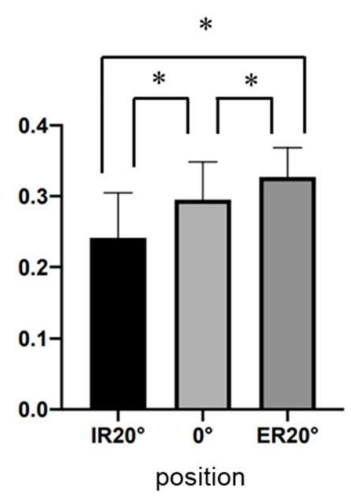

A: Side AB

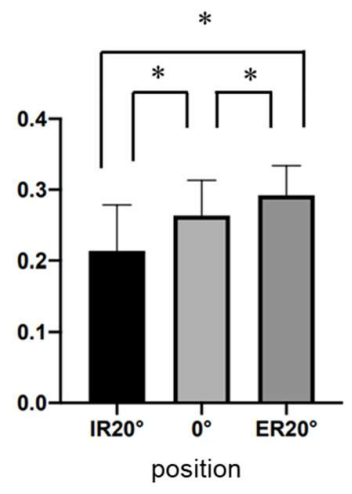

B: Side AC

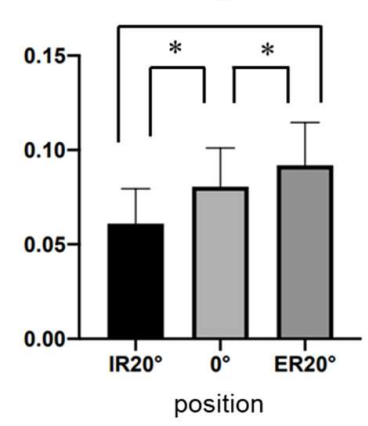

C: Side BC

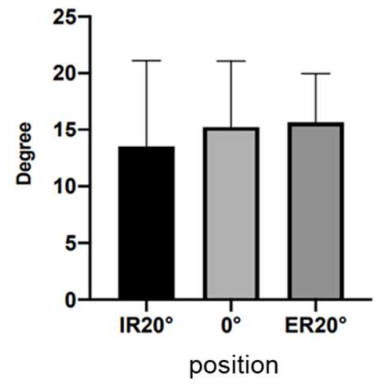

D: Angle A

Figure 2. Comparison of three sides and the angle of confluences of both heads of the biceps brachii at three shoulder positions. (A-C): Distances of Sides AB, AC, and BC. (D): Angle A. The measured distances of Sides AB, AC, and BC were standardized by dividing them by the humeral length. Angle A was calculated from Sides $A B, A C$, and $B C$ using the cosine theorem and then compared among the three shoulder positions. ER, external rotation; IR, internal rotation; ${ }^{*} p<0.0001$.

Table 3. Comparisons of Angle A and standardized Sides AB, AC, and BC by gender.

\begin{tabular}{cccc}
\hline & Male & Female & $p$-Value \\
\hline Angle A [degree] & & & \\
IR20 & $12.2 \pm 9.3$ & $14.1 \pm 7.1$ & 0.68 \\
$0^{\circ}$ & $14.9 \pm 5.4$ & $15.4 \pm 6.3$ & 0.86 \\
ER20 & $15.3 \pm 4.5$ & $15.8 \pm 4.4$ & 0.82 \\
\hline Side AB (standardized) & & & \\
IR20 & $0.23 \pm 0.04$ & $0.25 \pm 0.07$ & 0.47 \\
$0^{\circ}$ & $0.28 \pm 0.02$ & $0.30 \pm 0.06$ & 0.20 \\
ER20 & $0.31 \pm 0.01$ & $0.34 \pm 0.05$ & 0.08 \\
Side AC (standardized) & & & \\
IR20 & $0.20 \pm 0.05$ & $0.22 \pm 0.07$ & 0.48 \\
$0^{\circ}$ & $0.24 \pm 0.03$ & $0.27 \pm 0.05$ & 0.17 \\
ER20 & $0.28 \pm 0.02$ & $0.30 \pm 0.05$ & 0.18 \\
\hline Side BC (standardized) & & & \\
IR20 & $0.05 \pm 0.02$ & $0.06 \pm 0.02$ & 0.31 \\
$0^{\circ}$ & $0.07 \pm 0.02$ & $0.08 \pm 0.02$ & 0.40 \\
ER20 & $0.09 \pm 0.02$ & $0.10 \pm 0.02$ & 0.36 \\
\hline
\end{tabular}

The measured distances of Sides $\mathrm{AB}, \mathrm{AC}$, and $\mathrm{BC}$ were standardized by dividing humeral length. Data are presented as mean \pm standard deviation. ER, external rotation; IR, internal rotation. 


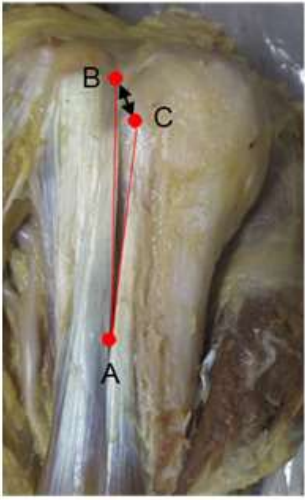

IR20

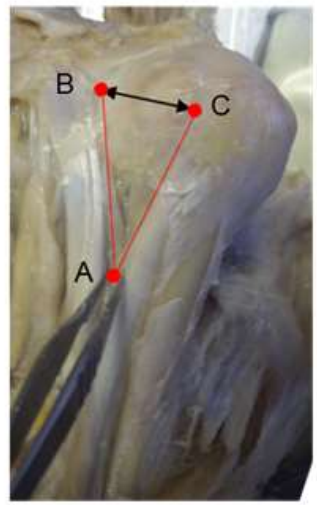

IR20

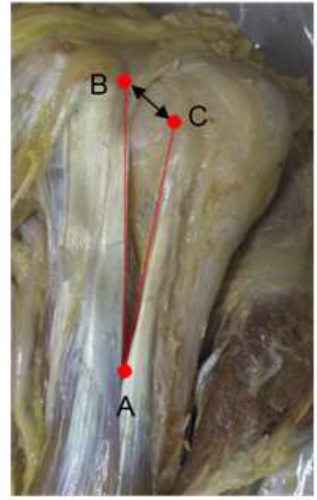

$0^{\circ}$

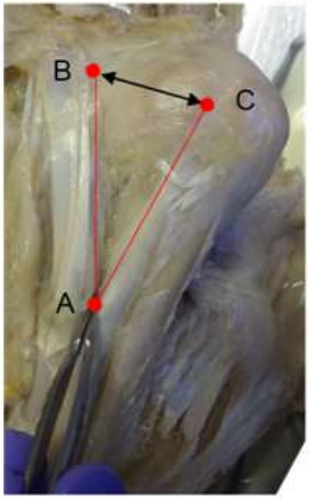

$0^{\circ}$

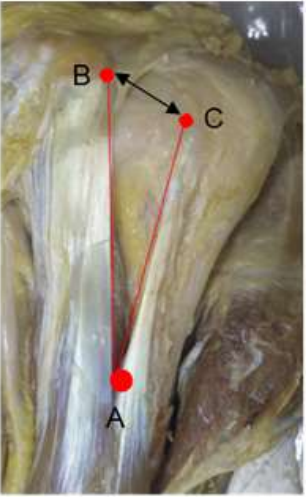

$\mathrm{ER} 20^{\circ}$

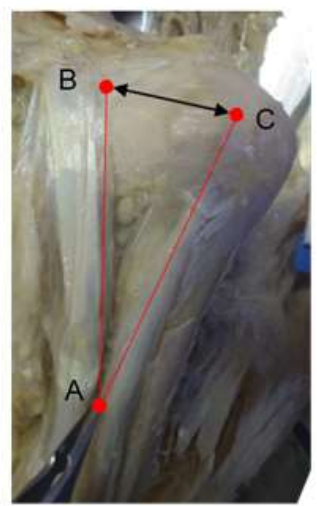

$\mathrm{ER} 20^{\circ}$

Figure 3. Photograph of the angle of confluence between the LHBB and SHBB (Angle A). In the case shown in the upper panels, Angle A increases during ER. In contrast, in the case shown in the lower panels, Angle A decreases during ER. ER, external rotation; IR, internal rotation; LHBB, long head of the biceps brachii; SHBB, short head of the biceps brachii.

\section{Angle A}

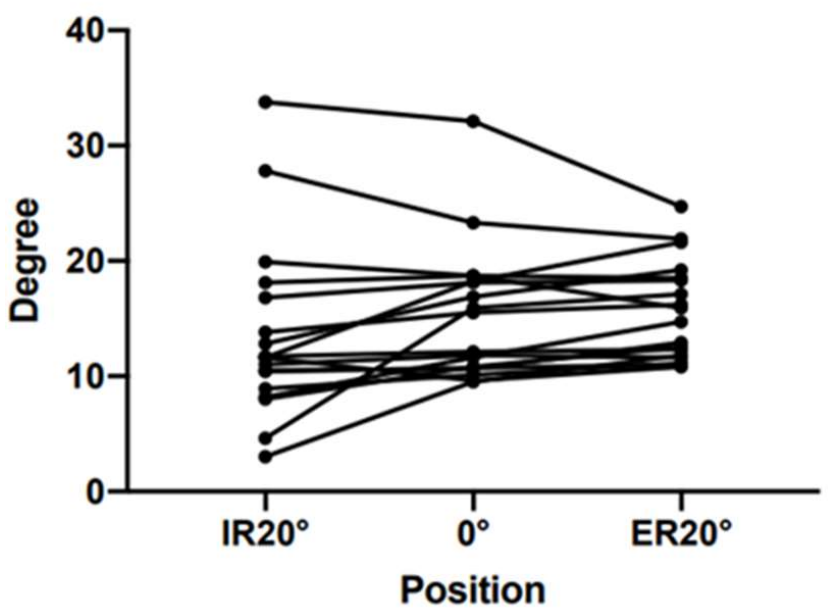

Figure 4. Distribution of Angle A in the three shoulder positions. ER, external rotation; IR, internal rotation. 


\section{Discussion}

Prior to this study, we hypothesized that the confluence of the SHBB and LHBB would remain fixed, but that the angle between them would increase during ER. However, our findings demonstrate that the angle between the LHBB and SHBB (Angle A) did not increase uniformly and tended to vary, more during IR than during ER. When angles were large at IR, Angle A tended to decrease towards ER, while small angles at IR tended to increase towards ER. These results suggest that this angle may converge in a certain range towards ER. It is particularly interesting to note that the position of the LHBB and SHBB confluence shifted distally during ER. In other words, the distance between the LHBB and SHBB upon shoulder rotation does not depend on the angle of their confluence, but rather on the movement of the confluence point.

The main actions of the biceps brachii are supination of the forearm and flexion of the elbow joint. Therefore, most studies on the muscle action of the biceps brachii have focused on the elbow joint and forearm motion [12,13]. Nevertheless, based on its anatomy, the biceps brachii functions as a flexor and an abductor of the shoulder joint $[1,14,15]$. Sakurai et al. [15] indicated that the LHBB can act as a humeral head stabilizer in the superior and anterior directions. Previous cadaver studies on the stabilizing effect of the LHBB at the shoulder joint have revealed that the LHBB contributes to shoulder joint stability when the biceps brachii acts as a flexor of the elbow joint [5,6]. Kumar et al. [6] investigated the distance between the acromion and the humeral head by applying weighted loads to the biceps brachii muscle in a cadaver study. Their results showed that the distance between the acromion and the humeral head significantly decreased during tension of the SHBB, while it did not change during tension of the LHBB or of both heads simultaneously. In addition, they reported that severing the tendon of the LHBB while both heads were tensed caused a significant upward migration of the humeral head [6]. In previous discussions on the stabilizing effect of the biceps brachii muscle on the shoulder joint, it was assumed that only the LHBB contributes to shoulder joint stabilization. However, based on Kumar et al.'s study, it seems that for the biceps brachii to contribute to shoulder stabilization, appropriate tension must be applied to both the LHBB and SHBB. In other words, it can be inferred that the SHBB also plays a role in stabilizing the shoulder joint, although this has not been clearly demonstrated. In support of this hypothesis, only scattered reports on rupture of the SHBB and its treatment are available [16-18].

Using radiographic images, Nobuhara [19] demonstrated that the humeral head inevitably moves upward due to the reduced stabilizing function of the LHBB during IR, whereas during ER, it clearly moves downward due to the stabilizing function of the LHBB. This phenomenon is generally interpreted from the positional relationship between the humeral head and LHBB, in which the LHBB passes over the humeral head during ER. Although the tension applied to the entire biceps brachii including the SHBB has not been considered, it is necessary to analyze the contributing factors to understand the stabilization mechanism of the LHBB during ER.

In the present study, during ER from IR $20^{\circ}$ to ER $20^{\circ}$, the distance between the LHBB and SHBB (Side BC) increased by approximately $9 \mathrm{~mm}$. The confluence of both heads moved distally at both sides of the SHBB and LHBB. That is, the outward movement of the LHBB during ER caused both heads to dissociate in the proximal part of the biceps brachii and the confluence to move distally in the distal part. This suggests that a tension that is different from the contraction force is added to the biceps brachii. During ER, the outward movement of the SHBB is smaller than that of the LHBB. The action of the force generated when the LHBB moves outward is thought to generate tension in the distal direction of the LHBB and SHBB (Figure 5). In fact, during the measurements, both heads were tensed during ER. This force is thought to be caused by the fact that the biceps brachii has two heads and the attachment of the SHBB is stable on the CP. In addition, considering that the angle of the confluence of both heads is concentrated at an angle of approximately $20^{\circ}$, the SHBB may have a tension control system that controls the outward movement of the LHBB during shoulder rotation. In case of conditions, such as biceps brachii elongation 
and adhesion, that prevent movement of the confluence of both heads, the damper function of the SHBB's may fail, and the stabilization of the shoulder joint by the LHBB may be inhibited. Moreover, based on the results of the present study, it can be predicted that the ER of the shoulder joint will produce a force that pulls the tendon of the SHBB distally. Therefore, for example, in the rehabilitation protocol after fixation of $\mathrm{CP}$ fractures, the motion of ER of the shoulder joint should be considered.

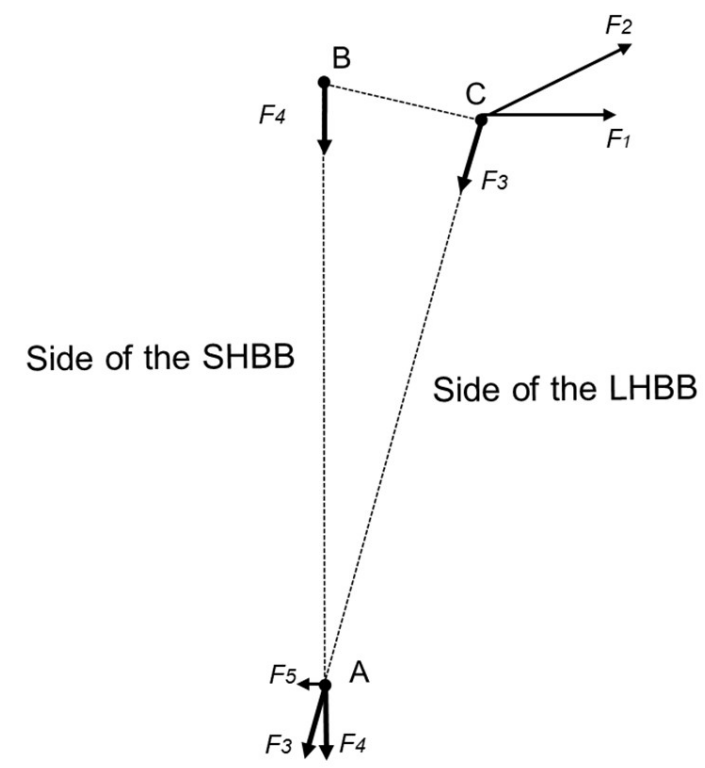

Figure 5. The forces that act on the biceps brachii tendons during external rotation. A: Confluence between the LHBB and SHBB. B: Lateral side of the SHBB at the top of the coracoid process level. C: Medial side of the LHBB tendon at the top of the lesser tubercle level. F1: Outward force of the LHBB due to external rotation. F2: Component force of F1. F3: Component force of F1; tension in the distal direction applied to the LHBB. F4: Component force of F3; tension in the distal direction applied to the SHBB. F5: Component force of F3. LHBB, long head of biceps brachii; SHBB, short head of biceps brachii.

Regarding the effect of gender on the range of motion of the shoulder joint, it has been reported that females have a greater range of motion than that of males $[20,21]$. However, in this study, there was no effect of gender on the morphological changes of the biceps brachii during shoulder rotation. This may be due to the effect of specific the range of motion used in this study (IR20 $20^{\circ}$ to $\left.20^{\circ}\right)$. In addition, some anatomical studies on the rotational range of motion of the shoulder joint have reported that the retroversion of the humeral head affects the external rotational range of motion of the shoulder joint [22,23]. However, we were not able to examine these in the present study. In light of these factors, we believe that future research should be based on the anatomical characteristics of each sex.

There are some limitations to this study. First, since it is a cadaver study, the effects in vivo are unknown and the effect of formalin fixation is unclear. Second, it is not possible to reproduce the afferent contraction of the biceps brachii. Third, the sample size was small. Fourth, there was no consideration of inter-rater and intra-rater variability due to a single measurement to eliminate the muscle stretching effect of the measurement. Finally, because this was an anatomical studies, aging process was not considered. In the future, it will be necessary to investigate the details of the tension applied to the biceps brachii and how it affects this muscle in vivo.

\section{Conclusions}

This study revealed the morphological changes occurring during shoulder rotation, namely the separation between the LHBB and SHBB and the confluence of both heads 
moving distally. This change may contribute to the stabilization of the shoulder joint. This information may be important for shoulder surgery and rehabilitation of associated injuries.

Author Contributions: Conceptualization, S.K. and S.H.; data curation, S.K., S.H., R.T., D.K., N.Q. and K.S. (Kaori Suyama); formal analysis, S.K., S.H., R.T., D.K., N.Q. and K.S. (Kaori Suyama); funding acquisition, S.H.; investigation, S.K.; methodology, S.K. and S.H.; project administration, S.H.; resources, K.S. (Kou Sakabe); supervision, K.S. (Kou Sakabe); visualization, S.K.; writingoriginal draft, S.K. and S.H.; writing-review and editing, R.T., D.K., N.Q., K.S. (Kaori Suyama) and K.S. (Kou Sakabe). All authors have read and agreed to the published version of the manuscript.

Funding: This work was supported by a Grant-in-Aid for Scientific Research (JSPS KAKENHI Grant Number JP17K01584).

Institutional Review Board Statement: This report complies with the research guidelines of the Japanese Association of Anatomists. This study was conducted under the approval of the Ethics Committee of Tokai University School of Medicine (Ethics Review Number 17R-307).

Informed Consent Statement: The cadavers used in this study were donated (Tokai Daigaku Kentai No Kai) for education or research. Informed consent was obtained from the antemortem person, Tokai Daigaku Kentai No Kai.

Acknowledgments: The authors thank Hayato Terayama, Noriyuki Kosemura, Kyoko Endo, and Yuko Furuya (of Tokai University School of Medicine, Kanagawa, Japan) for the excellent technical and secretarial support. Finally, the authors give their heart-felt appreciation to the body donors and their families.

Conflicts of Interest: The authors declare no conflict of interests. The authors, their immediate families, and any research foundations with which they are affiliated have not received any financial payments or other benefits from any commercial entity related to the subject of this article.

\section{References}

1. Gray, H.L.; Williams, P.L.; Bannister, L.H.E. Gray's Anatomy: The Anatomical Basis of Medicine and Surgery, 38th ed.; Churchill Livingstone/Elsevier: New York, NY, USA, 1995.

2. Tubbs, R.S.; Shoja, M.M.; Loukas, M. Bergman's Comprehensive Encyclopedia of Human Anatomic Variation; John Wiley \& Sons: Hoboken, NJ, USA, 2016.

3. Hollinshead, W.H. Anatomy for Surgeons, Volume 3: The Back and Limbs, 2nd ed.; Evanston and London, Harper \& Row Publishers: New York, NY, USA, 1969.

4. Kawakami, K.; Isogai, K. Anatomy and Surface Anatomy of Muscles, 2nd ed.; Daihokaku: Kumamoto, Japan, 2013. (In Japanese)

5. Itoi, E.; Motzkin, N.E.; Morrey, B.F.; An, K.N. Stabilizing function of the long head of the biceps in the hanging arm position. J. Shoulder Elbow Surg. 1994, 3, 135-142. [CrossRef]

6. Kumar, V.P.; Satku, K.; Balasubramaniam, P. The role of the long head of biceps brachii in the stabilization of the head of the humerus. Clin. Orthop. Relat. Res. 1989, 244, 172-175. [CrossRef]

7. Rodosky, M.W.; Harner, C.D.; Fu, F.H. The role of the long head of the biceps muscle and superior glenoid labrum in anterior stability of the shoulder. Am. J. Sports Med. 1994, 22, 121-130. [CrossRef] [PubMed]

8. Chen, C.H.; Chen, C.H.; Chang, C.H.; Su, C.I.; Wang, K.C.; Wang, I.C.; Liu, H.T.; Yu, C.M.; Hsu, K.Y. Classification and analysis of pathology of the long head of the biceps tendon in complete rotator cuff tears. Chang. Gung Med. J. 2012, 35, 263-270. [CrossRef] [PubMed]

9. Kido, T.; Itoi, E.; Sano, A.; Urayama, M.; Sato, K. The depressor function of biceps on the head of the humerus in shoulders with tear of the rotator cuff. J. Bone Joint Surg. Br. 2000, 82, 416-419. [CrossRef] [PubMed]

10. Mehta, S.K.; Teefey, S.A.; Middleton, W.; Steger-May, K.; Sefko, J.A.; Keener, J.D. Prevalence and risk factors for development of subscapularis and biceps pathology in shoulders with degenerative rotator cuff disease: A prospective cohort evaluation. J. Shoulder Elbow Surg. 2020, 29, 451-458. [CrossRef] [PubMed]

11. Nakagawa, Y.; Ozaki, J.; Sakurai, G.; Masuhara, K. A morphological and histological study of the long head of biceps brachii in torn rotator cuff shoulders. Katakansetu 1989, 13, 260-264.

12. Jarrett, C.D.; Weir, D.M.; Stuffmann, E.S.; Jain, S.; Miller, M.C.; Schmidt, C.C. Anatomic and biomechanical analysis of the short and long head components of the distal biceps tendon. J. Shoulder Elbow Surg. 2012, 21, 942-948. [CrossRef] [PubMed]

13. Pérot, C.; André, L.; Dupont, L.; Vanhoutte, C. Relative contributions of the long and short heads of the biceps brachii during single or dual isometric tasks. J. Electromyogr. Kinesiol. 1996, 6, 3-11. [CrossRef]

14. Kendall, F.P.; McCreary, E.K.; Provance, P.G.; Rodgers, M.M.; Romani, W.A. Muscles: Testing and Function with Posture and Pain, 4th ed.; Lippincott Williams \& Wilkins: Philadelphia, PA, USA, 1993. 
15. Sakurai, G.; Ozaki, J.; Tomita, Y.; Nishimoto, K.; Tamai, S. Electromyographic analysis of shoulder joint function of the biceps brachii muscle during isometric contraction. Clin. Orthop. Relat. Res. 1998, 354, 123-131. [CrossRef] [PubMed]

16. Fox, H.M.; Lunn, K.N.; Stewart, C.M.; Kanj, W.W.; Warner, J.J.P.; Chen, N.C. Rupture of the short head of the biceps brachii and coracobrachialis tendon: Repair with semitendinosus allograft. J. Shoulder Elbow Surg. 2020, 29, e350-e356. [CrossRef] [PubMed]

17. Moon, E.S.; Kim, M.S.; Kong, I.K. Traumatic isolated closed rupture of the short head of biceps brachii in military paratrooper. Knee Surg. Sports Traumatol. Arthrosc. 2010, 18, 1759-1761. [CrossRef] [PubMed]

18. Simon, M.; Lutter, C.; Schöffl, V. Rupture of the short head of the biceps brachii muscles belly caused by a rock-climbing accident. Wilderness Environ. Med. 2020, 31, 327-331. [CrossRef] [PubMed]

19. Nobuhara, K. The Shoulder: Its Function and Clinical Aspects, 4th ed.; Igaku-Shoin: Tokyo, Japan, 2012. (In Japanese)

20. Barnes, C.J.; Van Steyn, S.J.; Fischer, R.A. The effects of age, sex, and shoulder dominance on range of motion of the shoulder. J. Shoulder Elbow Surg. 2001, 10, 242-246. [CrossRef] [PubMed]

21. Vairo, G.L.; Duffey, M.L.; Owens, B.D.; Cameron, K.L. Clinical descriptive measures of shoulder range of motion for a healthy, young and physically active cohort. Sports Med. Arthrosc. Rehabil. Ther. Technol. 2012, 4, 33. [CrossRef] [PubMed]

22. Kronberg, M.; Broström, L.A.; Söderlund, V. Retroversion of the humeral head in the normal shoulder and its relationship to the normal range of motion. Clin. Orthop. Relat. Res. 1990, 253, 113-117.

23. Osbahr, D.C.; Cannon, D.L.; Speer, K.P. Retroversion of the humerus in the throwing shoulder of college baseball pitchers. Am. J. Sports Med. 2002, 3, 347-353. [CrossRef] [PubMed] 